\title{
Evaluation of a Neck-Shield for Use During Neurological Studies with a Whole-Body PET Scanner
}

\author{
C J Thompson ${ }^{1}$ Member IEEE, S Kecani ${ }^{2}$ and S Boelen ${ }^{3}$ \\ Montreal Neurological Institute ${ }^{1}$ and Dept. of Physics ${ }^{2}$ \\ McGill University, Montreal, and In.Co.De ${ }^{3}$ Pte. Claire QC Canada
}

\begin{abstract}
It is well known that the shielding against out-of-field scattered radiation is not very effective on whole body PET scanners operated with their slice-defining septa retracted. Additional shielding can be employed during neurological studies since the diameter of the head is much less than the field of view required for imaging the human torso. We have designed, built and tested (on the CTI HR+ PET scanner) a new lead-plate shielding system which mounts on the scanner's removable head-rest. We call this device the "NeuroShield". The outer diameter of the $9 \mathrm{~mm}$ thick lead plate is $550 \mathrm{~mm}$ which has a "U" shaped hole $220 \mathrm{~mm}$ in diameter. It is positioned just above the subject's shoulders. A moulded plastic coupling piece, produced by stereolithography, was designed to accommodate the complex shape of the headrest under the subject's neck, and provide a flat surface for mounting the lead plate. Our testing on human subjects involved comparing the prompt and random count rates and dead time during bolus-water activation studies. Scans on different subjects, (matched for age and sex) with no additional shielding, with the CTI Neurological Insert, and with the NeuroShield were compared. The random counts were reduced by a factor of 1.56 when using the permanent CTI Neurological Insert, and 1.44 using the NeuroShield. The NeuroShield is easily removed to allow whole body scanning. It can remain in place as the couch extends through the scanning tunnel in the gantry. Its main application will be at PET centres doing a mixture of whole body and neurological studies, where the CTI Neurological Insert cannot be installed permanently.
\end{abstract}

\section{INTRODUCTION}

\section{A. Shielding PET scanners from external activity.}

PET Scanners acquiring scans with their septa retracted are much more susceptible to excessive random count rates, scattered radiation and dead time due to activity outside the field of view (FOV). Several recent reports show the advantage of additional shielding to reduce these effects $[1,2,3]$. Grootoonk et al [1] showed the advantage of a permanent "Neurological insert" in the CTI HR ${ }^{+}$scanner, an option available with the instrument. Sossi [2] and Laforest [3] used lead shielding of the body regions axially beyond the field of view which were made for their situation. Hasegawa et al[4] have shown that in designing the shielding for use during neurological PET studies, the area of the shielding is more important than the thickness .

\section{B. Motivation for this device.}

At the time the CTI HR ${ }^{+}$scanner [5] at the Montreal Neurological Institute was purchased, the CTI "Neurological Insert" was not yet available. After its installation, our experience with it has been very positive and closely matches that of Grootoonk[1]. However, some studies, for example those requiring trans-cranial magnetic stimulation (TMS)[6] require equipment to be placed in the scanner which will not pass through the hole in the "Neurological Insert". During these studies the insert is removed so the quality of these studies is reduced due to higher random and scatter fractions. The original motivation to develop this device came from a desire to improve the quality of these studies. We are aware of many PET centres which perform a mix of neurological and other PET investigations which would benefit from shielding which could be easily removed, but provided similar performance than the "Neurological Insert".

\section{Materials ANd Methods} \section{A. Design and Development of the NeuroShield ${ }^{I}$.}

We have designed and built a new shielding system which we call the "NeuroShield". The principal novelty of our approach is that lead in the NeuroShield is attached to the head rest, not the gantry, and so moves with the couch. The advantage is that the diameter of the hole through which the subject's head must pass can be much smaller than when the lead is fixed. It also permits the couch to be fully extended through the gantry allowing full access to the head for stereotaxic placement of the (rather bulky) TMS stimulating coil.

The headrest on all recent CTI PET scanners ${ }^{2}$ including the EXACT HR+ is made from rigid plastic and part of its strength comes from its complex curvature. In order to mount the lead, a thermoplastic piece was designed to fit closely to contour of the headrest in the region between the couch top and the head support. This piece needed a plane vertical surface to which a cut lead plate is attached and four ribs between these two surfaces to provide structural support.

In order to design the coupling piece, the complete headrest was digitized on a numerically controlled milling machine to which a sensing probe was attached. The surface under the neck region was then used as one surface of a part,

\footnotetext{
${ }^{1}$ Scanwell Systems, Montreal Canada

${ }^{2}$ Private communication, Jonathan Frey, CTI
} 


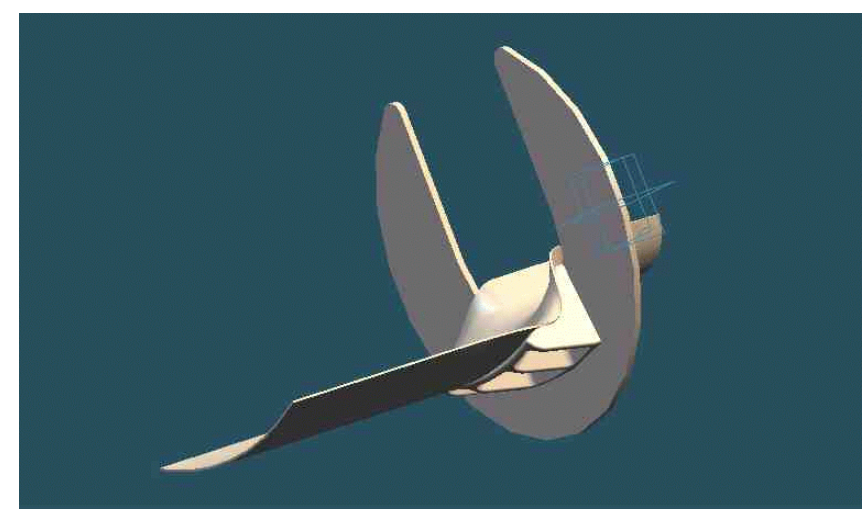

Figure 1 NeuroShield, head-rest and plastic coupling piece as rendered by "Solid Edge" 3D design program.

which was designed using the program "Solid Edge ${ }^{3}$ ".

A solid object was made by stereo-lithography. This process entails shining a modulated ultraviolet light beam on the surface of a resin bath in which the bottom is slowly lowered. The light cures the resin creating a solid object which is suitable for casting. From this a casting mould was made and the plastic resin coupling part was produced-colour matched to the scanner. The shielding itself is made from a 10 mm thick lead: antimony (95:5) allow which is much harder than pure lead. The lead plate was also designed with "Solid Edge" and cut to shape using a water jet. The plastic coupling piece requires no further finishing, but the lead was cleaned, sanded and painted to match the scanner. The fully assembled design is shown in Fig. 1, and the NeuroShield installed in the scanner is shown in Fig. 2.

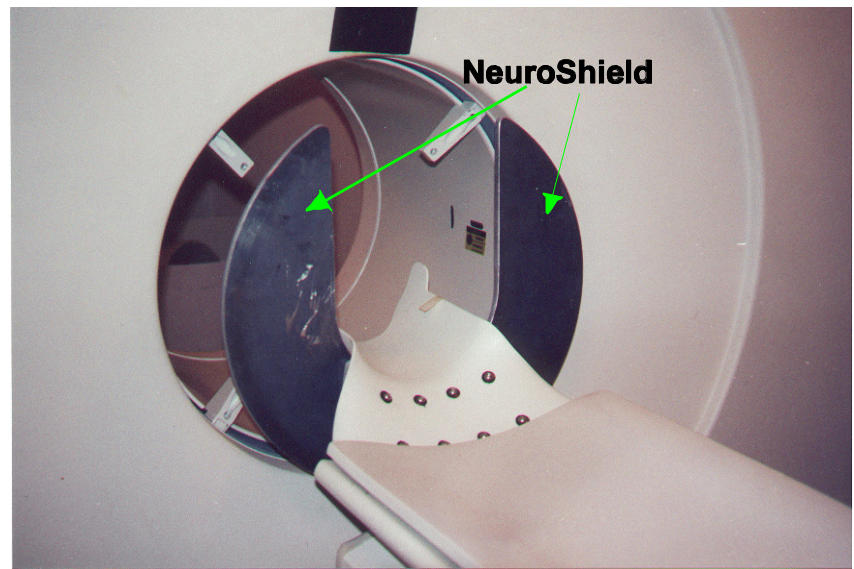

Figure 2 NeuroShield before painting ( $U$ shaped lead plate attached to head rest) in CTI scanner.

The headrest is attached to the scanner couch with two Velcroß strips along the entire $80 \mathrm{~cm}$ length which conforms to the couch contour. The lead piece is only $10 \mathrm{~cm}$ from the end of the couch, so even though the centre of gravity is well beyond the end of the couch the Velcro prevents it falling off. The NeuroShield is removed by peeling the Velcro starting at the end farthest from the head and lifting it off. After a few

\footnotetext{
${ }^{3}$ Unigraphics Solutions, Maryland Heights, MO
}

Table 1 Comparison of physical properties of CTI Neurological Insert and NeuroShield

\begin{tabular}{|c|c|c|}
\hline & $\begin{array}{c}\text { CTI Neuro- } \\
\text { Insert }\end{array}$ & NeuroShield \\
\hline $\begin{array}{c}\text { Outer } \\
\text { Diameter }\end{array}$ & $600 \mathrm{~mm}$ & $550 \mathrm{~mm}$ \\
\hline Inner Diameter & $400 \mathrm{~mm}$ & $220 \mathrm{~mm}$ \\
\hline Thickness & $9 \mathrm{~mm}$ & $9 \mathrm{~mm}$ \\
\hline Mass & $22.5 \mathrm{~kg}$ & $20.2 \mathrm{~kg}$ \\
\hline Area & $197 \mathrm{~cm}^{2}$ & $176 \mathrm{~cm}^{2}$ \\
\hline Attachment & Gantry & Headrest \\
\hline Removable & No & Yes \\
\hline $\begin{array}{c}\text { Whole body } \\
\text { scans? }\end{array}$ & No & Yes \\
\hline
\end{tabular}

studies have been performed, the Velcro is quite firmly set and it becomes more difficult to remove.

\section{B. Phantom Studies with a Point Source.}

Phantom studies were performed to measure the shielding effectiveness. For these studies a $20 \mathrm{~cm}$ diameter, 20 cm long flood calibration phantom containing $1.7 \mathrm{KBq}$ of ${ }^{68} \mathrm{Ge}$ was placed centrally in the scanner on the headrest. A 1100 $\mathrm{MBq}{ }^{137} \mathrm{Cs}$ source was mounted on a long rod and attached to a translation stage with a $30 \mathrm{~cm}$ travel. The source was advanced stepwise in $15 \mathrm{~mm}(\mathrm{v}=5 \mathrm{~mm} / \mathrm{sec})$ increments with a delay of 30 seconds at each step until it touched the surface of the stationary source. The experiment was performed with no shielding, and later with either the CTI Neurological insert or the NeuroShield in place. The upper level discriminator was set at $850 \mathrm{keV}$ to demonstrate the effect on the random count rate. An 600-second scan was performed for each shielding condition.

During each scan, the count-rates for prompts, randoms and dead time were recorded in the "rates-file" which can be produced on request during scans. The stop and go motion of the source driven by the translation stage produced a stair-case time-cont-rate curve which was compared for each condition.

\section{Count-Rates from Scans on Human Subjects}

During typical water bolus activation studies, 12 separate injections of $370 \mathrm{MBq}$ are used for separate scans and activation conditions. Rather than perform a new set of PET studies on the same subject with different shielding conditions, we chose to analyse data from similar subjects. Subjects whose data was used for this study were selected by different investigators in various protocols. These protocols were all approved by the MNI Research Ethics Committee and 
all subjects are required to give informed consent and provide a brief medical history. From these histories approximately age and sex matched subjects were chosen. The group of subjects who were scanned without additional shielding was used as a reference group since we have very few, of these. Subjects in this group, (M:3, F:5 age 27 y o) were all healthy non-smokers. Data from 6 clinical studies and 7 non-smoking volunteers (M:6, F7 age 33.8 y o) performed before the NeuroShield was installed, and a similar group (M:4, F6 age 46.8 y o) after the NeuroShield was installed in July 2000.

A "rates-file" is produced during bolus water studies which provides continuous information on the total (true + scattered + random) prompt counts, the delayed counts, dead time, frame number, etc, every second.

These rates files were processed to identify the point of greatest positive slope in the prompt count-rate by detecting the maximum difference between two contiguous sets of five consecutive points. The 12 curves were then averaged after time-skewing each scan for each subject. The time-aligned average scan rates file is saved for each subject. The standard deviation was calculated for all points and displayed for every eighth point to ensure that the scans were consistent and hence typical of that scanning condition. In some cases one scan file was removed from the set, if the data was very different from the other scans indicating a poor injection or other error in the scan protocol. The error bars in the figures below show the standard deviation of the count-rates at every eighth point after the time skew correction, to demonstrate that the count-rates during the scanning time are quite consistent for each subject The shape of these curves varies considerably from subject to subject due to variability in lung and cardiac function.

\section{Comparison of Images with the NeuroShield}

Since we did not perform any scans specifically to compare the various shielding conditions in individual subjects, it was not possible to compare image quality. In order to demonstrate that there are no apparent artefacts due to the asymmetry of the shielding, scans from two subjects who are sisters were aged 78 and 79 were compared. One of these was being studied for Alzheimer's disease, and the other was healthy and volunteered in a protocol to which elderly healthy subjects are recruited. Images through the lateral ventricles from a baseline study were compared in both subjects and reviewed for peak-to valley ratio (to verify that the scatter correction was not impaired) and front to back asymmetry (to assess the effect of high random counts in the frontal region due to lack of shielding in frontal region).

\section{RESULTS}

\section{A. Physical Characteristics of the NeuroShield}

The physical factors: size, weight, area of the CTI neurological insert and the NeuroShield are compared in Table I. Since the headrest attaches the couch with Velcro®, it is not "part of the scanner" and can easily be removed. This also ensures that once set in place, it is quite firmly attached and

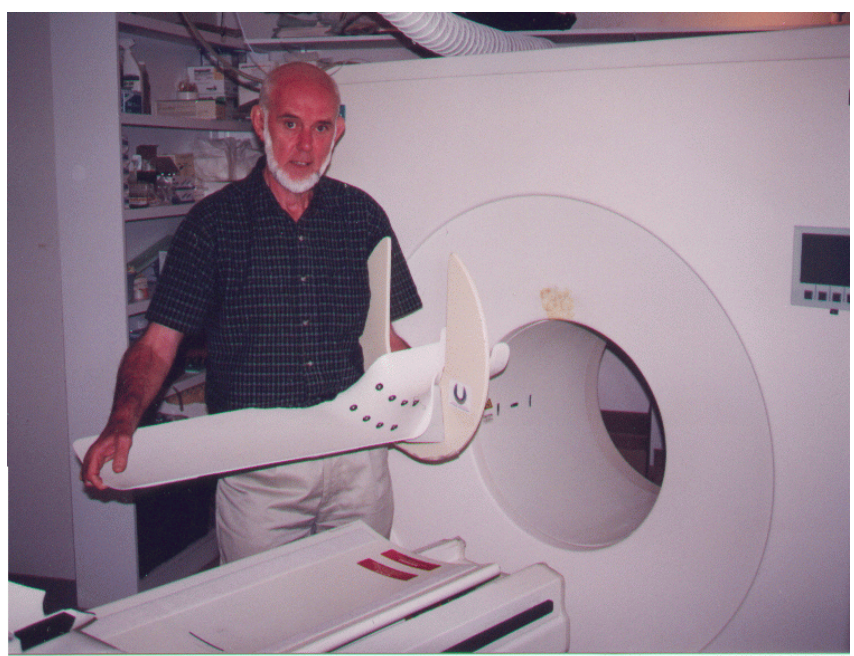

Figure 3 Illustration showing how NeuroShield is positioned on scanner's couch

could not accidentally fall off. Figure 3 shows the head-rest with the NeuroShield being placed on the couch. Figure 4 shows a subject being positioned for a scan.

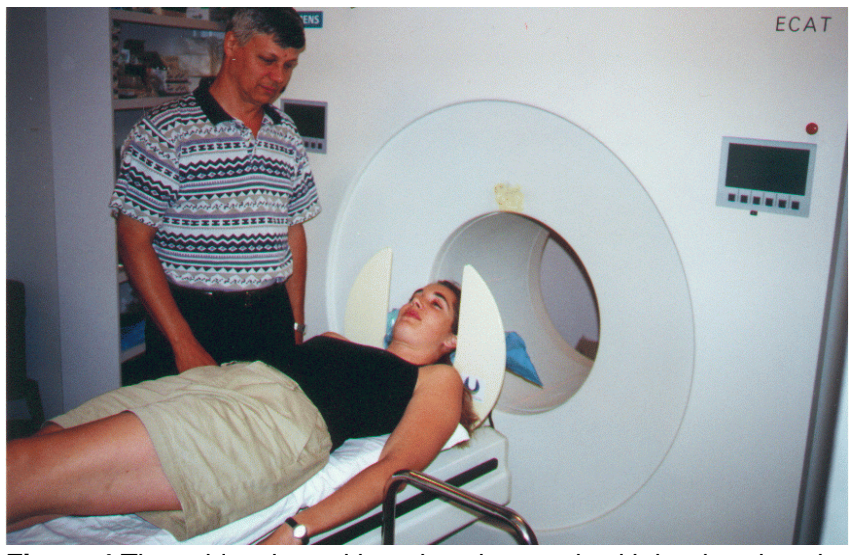

Figure 4 The subject is positioned on the couch with her head on the headrest, and her neck though the hole in the NeuroShield before the couch is moved into the gantry.

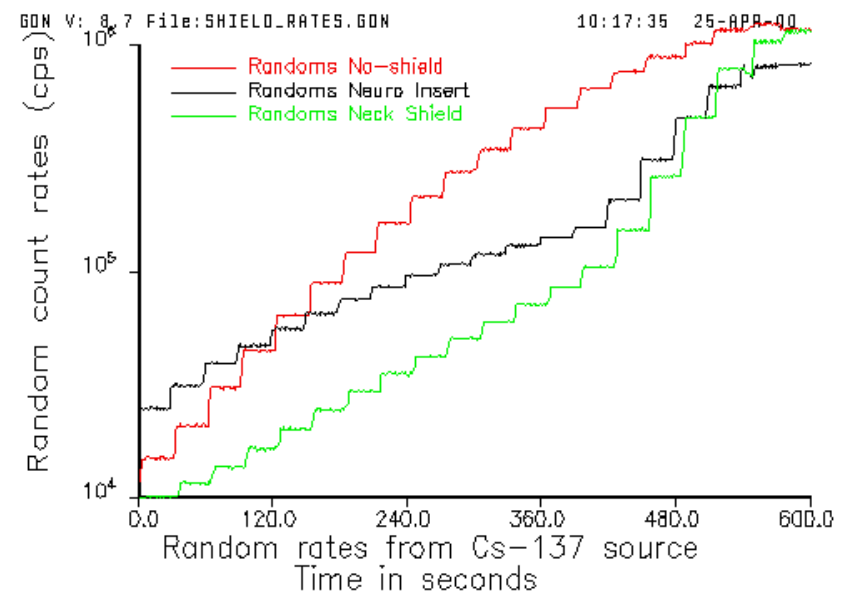

Figure 5 Random count rates from Cs-137 source which was moved from $30 \mathrm{~cm}$ beyond FOV in steps of $15 \mathrm{~mm}$ every 30 seconds towards scan field. During bolus water activation studies, random count-rates are in the range corresponding to the source's position between 4 and 5 minutes where the neck shield is very effective. 


\section{B. Phantom Studies.}

The results of the phantom studies are shown in Figure 5. The random count rates show clearly evident changes as the shielding is improved. Clearly both types of the additional shielding is most effective when the source is still some distance from the scan field. Since the aperture is smaller, the NeuroShield is superior to the CTI neurological insert until the source was closer to the scan field than the lead shielding (This occurs at a time of about 500 seconds in the graph.)

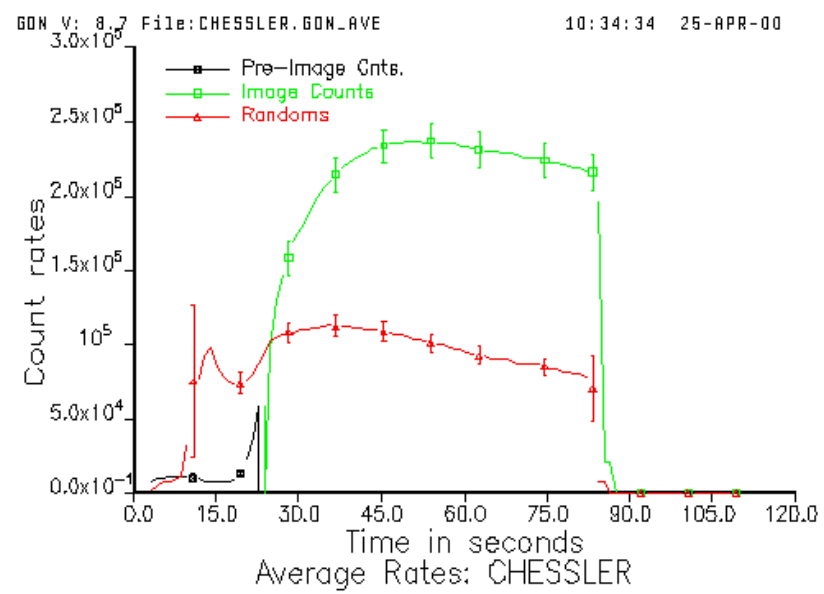

Figure 6 Count rates (prompts-delayed in green, randoms in red) before and during a scan with no additional shielding.

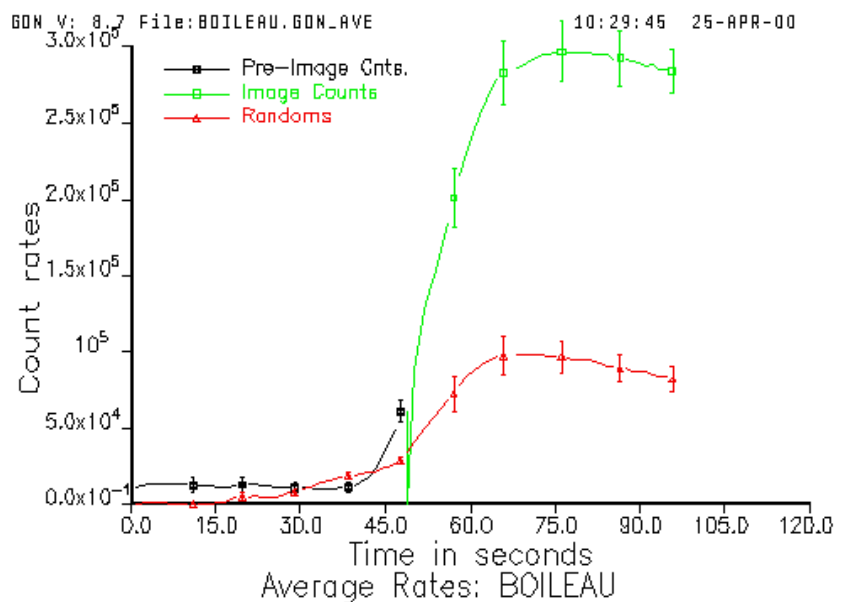

Figure 7 Count rates (prompts-delayed in green, randoms in red) before and during scan with Neuro-insert installed

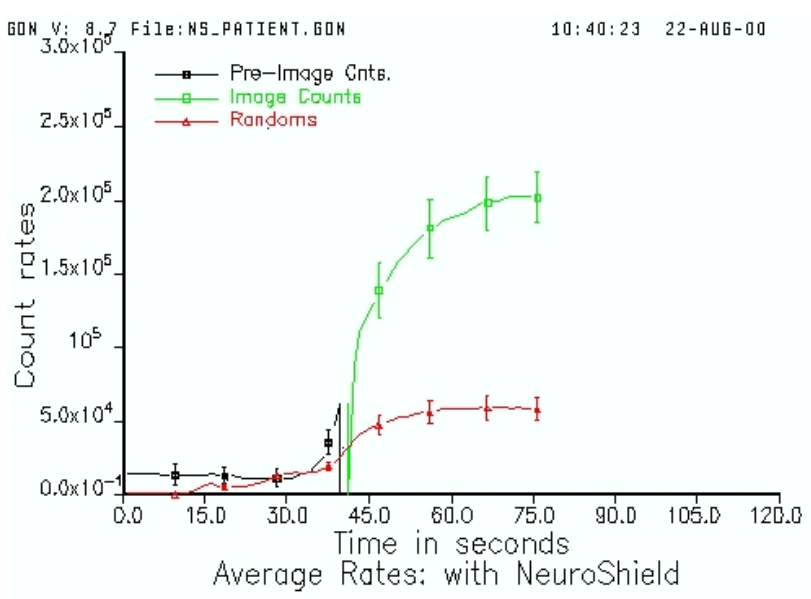

Figure 8 Count rates (prompts-delayed in green, randoms in red) before and during a scan using the NeuroShield.

\section{Count-Rates in Human Studies.}

Typical rates files (averaged over 10-12 injections in normal volunteers) are shown in figures 6 (no additional shielding), 7(with CTI neurological insert) and 8 with the NeuroShield). The results are summarized in Table II. The column designated "Prompt-randoms (kcps)" is the rate of true + scattered counts after subtracting the random count-rate. There is a significant reduction in the random count rate with either the CTI neurological insert or NeuroShield installed. The dead time is also lower by about $1.5 \%$ with both methods of shielding. The average prompt count rates are reduced as the shielding improves but the differences are less than one standard deviation. The scattered radiation from the body will be reduced by the shielding so one would expect the prompt count-rate to be less.

\section{Comparison of Images from Human Subjects}

Profiles through images through the lateral ventricles from two sisters one scanned with the CTI Neurological Insert, and the other scanned with the NeuroShield in place are shown in figures 9 and 10. There are no artefacts due to a relatively higher possibility of random counts being detected along lines of response through the frontal brain regions.

Table 2 Comparison of (prompt-random) and random count rates for three shielding configurations.

\begin{tabular}{|c|c|c|c|c|c|}
\hline & $\begin{array}{c}\text { \# of } \\
\text { subjects }\end{array}$ & $\begin{array}{c}\text { \# of } \\
\text { scans }\end{array}$ & $\begin{array}{c}\text { Prompt-randoms } \\
\text { (kcps) }\end{array}$ & $\begin{array}{c}\text { Randoms } \\
\%\end{array}$ & $\begin{array}{c}\text { Dead time } \\
\%\end{array}$ \\
\hline No Shielding & 8 & 96 & $217 \pm 58$ & $48.9 \pm 5.5 \%$ & $15.5 \pm 1.5 \%$ \\
\hline CTI Insert & 12 & 147 & $210 \pm 54$ & $31.2 \pm 4.2 \%$ & $14.1 \pm 2.2 \%$ \\
\hline NeuroShield & 7 & 92 & $196 \pm 42$ & $33.9 \pm 4.0 \%$ & $14.0 \pm 1.8 \%$ \\
\hline
\end{tabular}




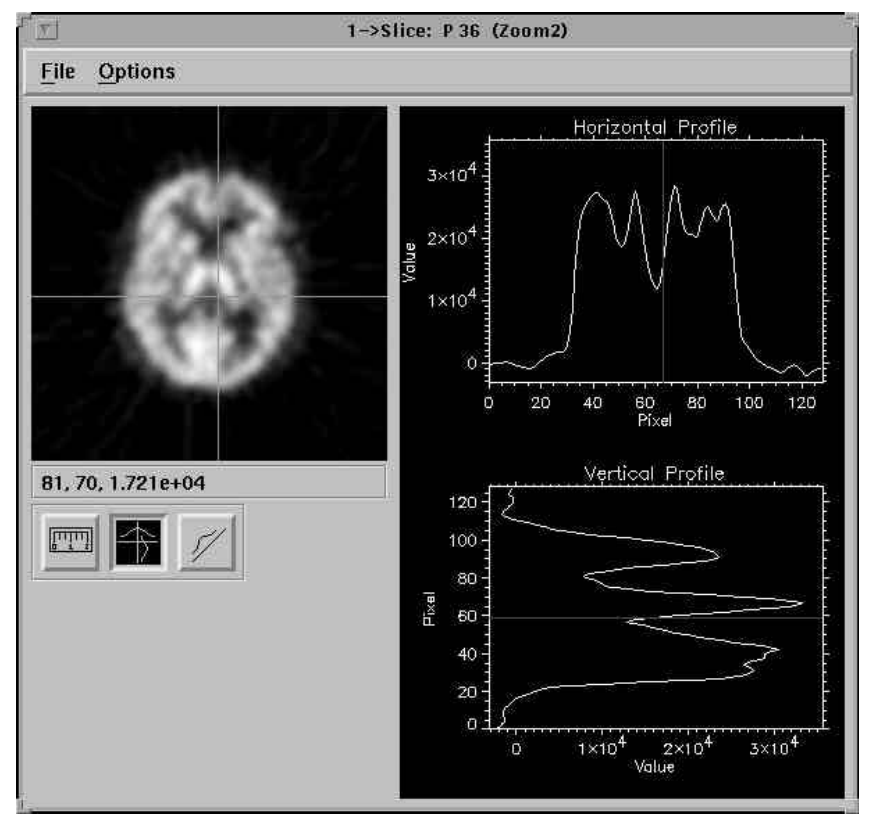

Figure 9 One slice through basal ganglia and profiles from subject scanned using CTI neurological insert.

\section{DiscuSSION}

Phantom studies show that NeuroShield is most effective in shielding when activity is in the range of $20-30 \mathrm{~cm}$ from scan field. As the source approaches the scan field, the detectors, especially those near the back for the scan field, have a direct line-of-sight view of the source so shielding is less effective. However the NeuroShield is very useful in shielding from activity in the lungs and heart where the out-offield activity is most concentrated.

Analysis of human studies show that the NeuroShield is effective, but not quite as good as CTI insert (although the difference is not statistically significant). In this study, the subjects are age matched, but the scans are not the same subjects. In order to test the shielding more thoroughly it

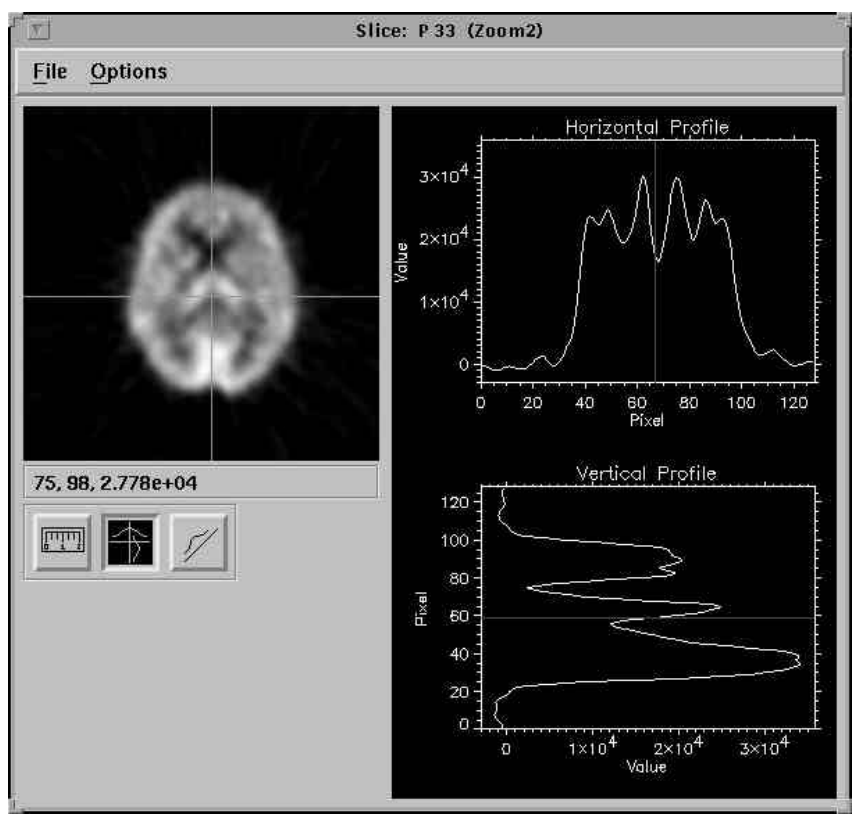

Figure 10 One slice through the basal ganglia and profiles from a subject scanned using the NeuroShield. would be necessary to scan the same subject with all three shielding conditions. However there is a difficulty to justify doing scans on human subjects solely to demonstrate efficacity of shielding.

The NeuroShield is easy to install and well accepted by patients, staff and researchers. The $22 \mathrm{~cm}$ wide vertical cutout does not pose any problems when the subjects are viewing a large screen video monitor, nor in their access to the touch sensitive surface sometimes used to provide feedback during scanning.. It has also been found very useful for the improved access to patient's head. Some researchers have attached a bite-bar to the NeuroShield to help maintain subject position. This has been found easier than clamping it to the headrest and it is more rigid.

There was concern about subject safety when the idea was originally proposed. However the quick-release used to unlock the couch drive is just as effective as before. With the CTI insert, there is a slight chance that the subject's head could collide with the shielding if their head is not restrained while the patient is being scanned. This is much less likely with the NeuroShield since the lead plate moves with the subject.

The headrest used in this scanner is the same one used in all recent CTI scanners, so the NeuroShield should work on any of these. The same concept could be adapted to other whole body PET scanners like the GE Advance®. It would probably be even more effective on systems with dual headed gamma cameras operating in coincidence since these have no fixed shielding on the detectors.

\section{Conclusions}

The NeuroShield has been shown to have similar shielding properties the than "permanent shielding" provided by CTI. It has the great advantage of being easily removable making it suitable for use in sites which do a mixture of PET scans during brain studies. It has been very well accepted by subjects, researchers and technical staff.

\section{ACKNOWLEDGEMENTS}

The development of novel PET instrumentation concepts and the Montreal Neurological Institute is supported by a grant from the National Science and Engineering Council of Canada (OGP-0036672) to Dr. Thompson. The materials for this project were provided through a group grant from the Medical Research Council of Canada to Dr. Alan Evans and collaborators. The data from human subjects was available as a result of studies done by several researchers at the MNI including Tomas Paus, Robert Zattore and Denise Kline. We would like to thank the technical staff of the McConnell Brain Imaging Centre especially Richard Fukasawa and Garry Sawchuck for their help and enthusiasm for this project.

\section{REFERENCES}

1. Grootoonk S, Fink G, Brun, T: "Impact of a NeuroInsert on Brain Scanning with the ECAT EXACT HR+ PET Scanner", Neuroimage 1996 vol 3 no 3 S25. Conf proceedings: 2nd Intl. Conf. on Functional Mapping of the Human Brain. 
2. Sossi V, Pointon B, Cohen P, Johnson R, Ruth T: "Effect of Shielding the Radioactivity Outside the Field of View on Image Quality in a Dual Head Coincidence Camera" IEEE 1999 Conference Record (M8-4)

3. R. Laforest, P. Cutler, M. Daube-Witherspoon: "Extra-Shielding for Improved Signal-to-Noise in 3D Whole-Body PET" IEEE 1999 Conference Record (M8-5)

4. Hasegawa T, Murayama H, Nakajima Y, Matsuura H, Wada Y: "A Study of External End-shields for PET" IEEE 1999 Conference Record (M10-090)

5. Moreno-Cantu J J, Thompson C J, Zattore R J: "Evaluation of the ECAT Exact HR+ 3D PET Scanner in O-15 Water Brain Activation Studies". IEEE Trans. Med. Imag. 17:6 979-985 (1998).

6. Paus T, Jech R, Thompson C J, Comeau R, Peters T M, Evans A C: "Trans-cranial Magnetic Stimulation During Positron Emission Tomography: A New Method for Studying Connectivity in the Human Cerebral Cortex" J Neuroscience 17(9): 3178-3184 (1997). 